\title{
Non-Perturbative 2 Particle Scattering Amplitudes in 2+1 Dimensional Quantum Gravity
}

\author{
G. 't Hooft
}

Institute for Theoretical Physics, Princetonplein 5, P.O. Box 80.006, NL-3508 TA Utrecht, The Netherlands

\begin{abstract}
A quantum theory for scalar particles interacting only gravitationally in $2+1$ dimensions is considered. Since there are no real gravitons the interaction is entirely topological. Nevertheless, there is non-trivial scattering. We show that the two-particle amplitude can be computed exactly. Although the complete "theory" is not well understood we suggest an approach towards formulating the $N$ particle problem.
\end{abstract}

\section{Introduction}

It is not known how to quantize gravity without running into infinity problems or topological contradictions such as the ones that are hampering our understanding of black holes. Even in $2+1$ dimensions quantum gravity is non-renormalizable. Yet there is reason to hope that a consistent formulation of a quantum theory can be given that yields classical $2+1$ dimensional gravity in the limit $\hbar \Rightarrow 0$. Our reason for thinking this is that in $2+1$ dimensions the gravitational interaction is entirely topological; there are no real gravitons, and the only degrees of freedom are whatever other particles are being introduced. Classically, the "interaction" is simple and beautiful [1]: every particle is surrounded by a space-time in the form of a cone. The conical singularity is at the world line of the particle, and the deficiency angle at this singularity can be defined to be equal to the particle's mass (we put Newton's constant equal to one). As a consequence, two particles passing each other at the right proceed in a direction slightly different from the one they choose when they pass each other at the left.

If we know for each particle at which side they pass each other particle then the classical scattering process is trivial to compute: they all continue in straight lines. If this is so simple, why then can't we "quantize" this system by attributing wave packets to these particles?

Trying to do just this, one discovers a difficulty. Particles in the wave packets are not well localized. This does not stop us from writing down one-particle wave equations on a cone, but the difficulty comes in writing down many-particle wave equations. Where exactly is the conical singularity produced by one particle in the space-time of another? 
The problem is to set up a Hilbert space of wave functions and their corresponding wave equations in the multi-dimensional, "multi-conical" spacetime spanned by the $N$ particle states. Although one does seem to hit some fundamental difficulties in trying to do this, it does not seem to be altogether impossible. Remarkably, we found that the two-particle sector of Hilbert space can be constructed unambiguously, and the scattering amplitude is unambiguous. The mathematics of this scattering is quite nice, it eventually amounts to nothing but wave mechanics on a cone.

A standard way to deal with wave mechanics on a cone is to diagonalize the angular momentum operator. Bessel functions with fractional indices result. Certainly one will be able to obtain the scattering amplitudes for colliding plane waves of particles this way [2], but we chose for a more direct method. Since we wanted to see how plane ingoing waves evolve into superpositions of plane outgoing waves we avoid the double expansions needed when working with Bessel functions, but construct the solution of the wave equation (with the given initial conditions) directly. As a bonus we then discover that, contrary to the classical case, particles can circle each other many times before parting (what is meant by this statement mathematically will become clear in the text). The importance of this latter observation is that it will make the more general $N$ particle case definitely much more difficult than the corresponding classical problem.

We believe that a more complete understanding of the system considered in this paper might provide us with important clues for handling quantum gravity in the real world. For instance, quantization of angular momentum (even though it is anomalous, see Sect. 4) in some respects seems to indicate that time itself is quantized. Quantization of time may also be suggested by observing that the total energy is limited to be either less than $2 \pi$ (for open systems), or equal to $4 \pi$ (when space-time is closed). Indeed it might be necessary to introduce a lattice for spacetime. In this paper, we will not expand any further on such speculations however.

Also not considered in this paper are any interactions other than the gravitational ones. But our suspicion that, since the total energy is bounded, infinities in loop integrations will be cut off in a natural way was a strong motivation for studying this system.

To achieve a consistent theory it is of importance to avoid the more standard methods of quantizing gravity as if it were a gauge theory [3]. Then namely one introduces both virtual gravitons and ghosts, all of which might have unlimited energies. What we are trying to do is first to consider the real degrees of freedom, which are just the spectator particles (whose non-gravitational interactions could be renormalizable or super-renormalizable), surrounded by a funny geometry. We then try to quantize these directly.

One consequence of our approach is that creation or annihilation of particles are not seen to occur. That perturbative $2+1$ dimensional gravity does predict creation and annihilation, as we will check in Sect. 8, reminds one of the fact that our understanding of the $N$ particle problem is very incomplete.

\section{Hilbert Space}

As stated in the Introduction, it will be difficult to set up a Hilbert space describing an $N$ particle configuration at a given instant $t$. Classically (that is, without 
quantum mechanics), the positions $\mathbf{x}_{i}(t)$ are well defined if all particles are connected to an observer by strings; the only features of these strings that are to be specified are the ways (left or right) along which they pass the other particles and each other. This then gives us an ordered set of coordinates, but there is clearly a redundancy, because the choice of the string paths was arbitrary. If the deficiency angles at all the conical singularities were specified we could precisely write down which sets of coordinates are equivalent, and set up our wave equations with their boundary conditions.

However, as we will now explain, the conical singularities depend on the positions, and the momenta of the particles. The best way to specify the singularity is to write down which element $P$ of the Poincaré group identifies points $(\mathbf{x}, t)$ having left going strings with points $\left(\mathbf{x}^{\prime}, t^{\prime}\right)$ having strings passing the particle along the right. For a spinless particle at rest at the origin this is

$$
(\mathbf{x}, t)=\left(\begin{array}{ccc}
\cos m & \sin m & 0 \\
-\sin m & \cos m & 0 \\
0 & 0 & 1
\end{array}\right)\left(\mathbf{x}^{\prime}, t^{\prime}\right)
$$

( $m$ is its mass) ${ }^{1}$ and for a moving particle going through the point $(a, 0)$,

$$
(\mathbf{x}, t)=L\left(\begin{array}{ccc}
\cos m & \sin m & 0 \\
-\sin m & \cos m & 0 \\
0 & 0 & 1
\end{array}\right) L^{-1}(\mathbf{x}-\mathbf{a}, t)+(\mathbf{a}, 0)
$$

where $L$ is the Lorentz transformation that gives the particle its specified momentum. For instance, if $\mathbf{p}$ is in the $x$-direction we have

$$
L=\left(\begin{array}{ccc}
\cosh \lambda & 0 & \sinh \lambda \\
0 & 1 & 0 \\
\sinh \lambda & 0 & \cosh \lambda
\end{array}\right)
$$

with $\lambda$ such that

$$
\left(\begin{array}{l}
p \\
0 \\
E
\end{array}\right)=L\left(\begin{array}{l}
0 \\
0 \\
m
\end{array}\right)
$$

Notice now that Eq. (2.2) contains both the particle's position a, and its momentum p via Eqs. (2.3) and (2.4). The difficulty mentioned in the Introduction is that these $\mathbf{a}$ and $\mathbf{p}$ do not commute.

It is not hard to verify [1] that if the total energy in the center of mass coordinates is $E$ and the total angular momentum is $l$, then the space-time surrounding the complete system is a piece of a "twisted" cone. The complete cone would have a singularity with deficiency angle $\delta \varphi$, and in addition a "twist" in the time direction: running on an equal time curve around the system one returns to

\footnotetext{
${ }^{1}$ In this paper units for mass, momentum and energy will be chosen as in Eq. (2.1). In more detailed calculations however it is often morc convenient to use $S U(1,1)$ matrices rather than the $S O(2,1)$ ones, in which case natural units differ by a factor 2 from ours
} 
the same region with a shift $\delta t$ in time. The absolute value of the shift $\delta t$ is equal to the angular momentum $l$. Careful analysis of the sign of $\delta t$ reveals that it is such that a rotation around the system in the same direction as the rotation that produces $l$ is associated with a shift backwards in time. One derives

$$
\delta \varphi=E ; \quad \delta t=-l .
$$

Strictly speaking Eqs. (2.5) can only be derived in systems for which $E$ and $l$ are small, because only in flat space-times the total energy and angular momentum are unambiguous. However it is obvious that in this particular case $\delta \varphi$ and $\delta t$ are additive and obey conservation laws; they are well defined regardless how large they are. Therefore it is natural to define energy and angular momentum through (2.5) in all cases.

Clearly, if $E<2 \pi$, the system sits in an infinite space-time. Let us concentrate on that case. Classically we then expect at $t \Rightarrow \pm \infty$ all particles to be infinitely far apart. Their velocities will all be directed radially inward at $t \Rightarrow-\infty$ and outward at $t \Rightarrow+\infty$. But now we can mimic this situation quantum mechanically, by attributing to these particles widely extended but still reasonably localized wave packets. Then their momenta are all well-defined, as well as the routing of all "strings" that we had to connect to these particles. Therefore we may still be able to define Hilbert spaces for the asymptotic states at $t \Rightarrow \pm \infty$. We may ask how a given in state evolves into certain out states.

Notice that these wave packets will be handled as if space-time were completely flat. The cusps that we should remove from space-time so as to turn it into the required multi-conical shape can all be defined to be pointed outwards, so that an observer situated closer to the interaction region will not notice this deviation from flatness. The strings mentioned before can all be drawn along straight lines connecting the particles with the observer. We caution that a precise formulation of unitarity and completeness is yet to be given and won't be easy, because the wave packets were crucial. It is definitely not allowed to simply expand these into plane waves because then the ordering problem for the strings reemerges. But we will now show that at least in the two particle case this approach is going to work just fine.

\section{The 2 Particle Sector}

Let us first consider the classical (=unquantized) 2 particle case. There is an observer at some far away but fixed point $\mathbf{0}$, linked to a fixed Lorentz frame. The in state consists of two particles, surrounded by a space-time as described in [1]. Both particles each form a pointlike singularity, which can be described as a conical singularity, as if an angular wedge with angle $\alpha$ ("deficiency angle") is removed from a flat space. In coordinates where a particle is at rest the corresponding deficiency angle equals the particle's mass $m$. The space-time surrounding the twoparticle system can be seen to correspond to a cone whose deficiency angle in the center of mass frame equals the total energy $E$, but in addition there is an extra piece of space-time in between the two particles removed.

We decide to describe the space-time surrounding our two particles by indicating their positions $\mathbf{x}_{1}(t)$ and $\mathbf{x}_{2}(t)$ in a flat coordinate frame, and drawing 


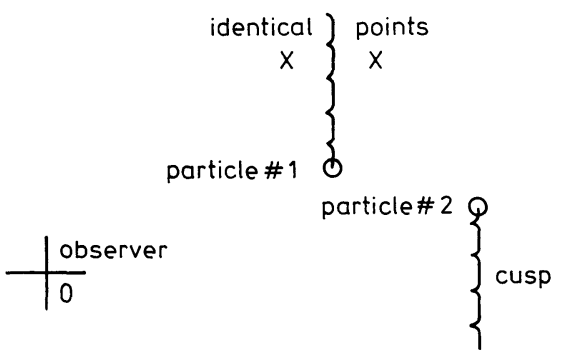

Fig. 1. The two particle scattering arrangement

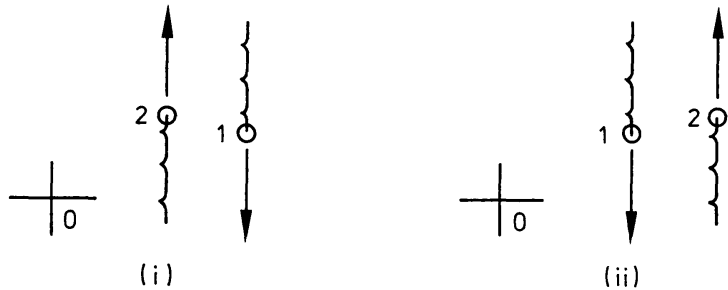

Fig. 2. The two scattering possibilities

their cusps pointed outwards. Their distance is

$$
\mathbf{r}(t) \equiv \mathbf{x}_{1}(t)-\mathbf{x}_{2}(t) .
$$

The cusps (the deficiency angles as well as their locations) are given by specifying the elements $P$ of the Poincare group that connect the flat sections of space-time when one follows a loop around the particle. In Fig. 1 we indicate the location of the cusps by wavy lines; one must bear in mind that if flat coordinates are used points close to a wavy line may have to be identified to corresponding points at the other side.

In the in state, $|\mathbf{r}|$ must be a decreasing function of $t$. There are now two possibilities: $\mathbf{r}$ may pass the origin either (i) at the left, or (ii) at the right. The vector $\mathbf{r}$ itself is linear in $t$, only if both particles and the observer do not cross any of the cusps. If they do then we must perform the relevant Poincaré transformation to see how $\mathbf{r}(t)$ continues.

It now becomes crucial that we should limit ourselves to the case that the observer 0 stays far away, so that we can exclude the possibility that the two particles pass $\mathbf{0}$ at opposite sides. Let us now also describe the out states such that the cusps are pointing outwards. The two possibilities are now indicated in Fig. 2.

We see that if we wish to rotate the cusps outward in the out states without crossing the particles then in case (i) cusp 2 crosses the observer 0 clockwise and in case (ii) it is cusp 1 that crosses $\mathbf{0}$ anticlockwise. Thus, in the two cases, the vector $\mathbf{r}(t)$, originally linear in $t$, is seen by the observer in the outgoing state as

$$
r_{(\mathrm{i})}=P_{2}\left(\begin{array}{l}
\mathbf{r} \\
t
\end{array}\right) \text { and } r_{(\mathrm{ii})}=P_{1}^{-1}\left(\begin{array}{l}
\mathbf{r} \\
t
\end{array}\right)
$$


respectively. Clearly,

$$
r_{(\mathrm{i})}=P_{2} P_{1} r_{(\mathrm{ii})} \text {. }
$$

We conclude that the $(2+1)$ vector $r$, which is the distance between the two particles, sits in a conical space whose deficiency angle is determined by the product $P_{2} P_{1}$. We note that in the center of mass coordinate frame, $P_{2} P_{1}$ is a rotation about the origin, over an angle which corresponds to the c.m. energy $E$, combined with a translation in time over a distance $\delta t$ equal to the total angular momentum $l$.

Note that the conical space spanned by the allowed values of the relative coordinate $\mathbf{r}$ has the same deficiency angle as the space-time surrounding the two particles, but it is an entirely different space. In particular there is no further excised region. The space-time surrounding the two particles would rather correspond to the configuration space for a third, very light particle. In the space-time surrounding the two particles the tip of the cone whose angle is $E$ is in the forbidden region and therefore not a real singularity. In the configuration space for $\mathbf{r}$ the tip, which is at the origin $\left(\mathbf{x}_{1}=\mathbf{x}_{2}\right)$, is a physically accessible point.

So now we know how the relative coordinate $r$ evolves in space-time. The coordinates of the center of mass, $R$, are even simpler. These can be defined as the location of those points (the "tip of the cone") for which the transformation $P_{2} P_{1}$ gives a pure time translation:

$$
P_{2} P_{1} R=R+l \hat{t},
$$

where $\hat{t}$ is the unit vector in the time direction. Clearly, these points $R$ always form a straight line. With respect to the observer $\mathbf{0}$, the center of mass coordinates $\mathbf{R}(t)$ occupy a flat space-time. There is a single cusp emanating from $\mathbf{R}(t)$ which we can choose to be pointing always away from $\mathbf{0}$.

This completes our description of the classical parameters. The center of mass coordinates $\mathbf{R}(t)$ evolve in a flat space-time, and the relative coordinates $\mathbf{r}(t)$ sit in a cone, whose deficiency angle equals the total c.m. energy $E$, but when going around the cone we must also make a time shift proportional to $l$.

The fact that the space-time for $\mathbf{r}(t)$ depends on $E$ and $l$ is a rather delicate feature of this system. It implies that we will have to describe scattering at a fixed and well determined value for $E$, an important limitation when we wish to turn to quantum mechanics. We do not have to keep $l$ fixed, as we will explain.

\section{Quantum Mechanics}

We choose the total energy to be less than $2 \pi$, so that there are asymptotic in and out regions. Since $\mathbf{R}(t)$ is trivial we may limit ourselves to the case $\mathbf{R}(t)=0$ and concentrate on $\mathbf{r}(t)$. First there is a subtle question concerning the time shift in the conical boundary condition.

To take the conical deficiency angle into account is easy. We consider the "looping operation": one particle is rotated around another over a complete loop and returns to its original position. The new configuration is identical to the old one except for the strings linked to the particles, which now follow a different route. 
Cones and other locally flat spaces can now be specified by plugging a non-trivial "loop operator" into the "looping boundary condition." Suppose we took a basis in which angular momentum is diagonalized:

$$
\psi=e^{i l \varphi} \psi_{l},
$$

then we have the boundary condition

$$
\psi_{\text {loop }} \equiv \psi(\varphi+2 \pi)=\psi(\varphi+\delta \varphi),
$$

with

$$
\delta \varphi=E .
$$

Clearly, this boundary condition can also be formulated as

$$
\psi_{\text {loop }}=e^{2 \pi i l} \psi=e^{i l E} \psi,
$$

and it leads to the revised quantization law for angular momentum:

$$
l=\frac{2 \pi m}{2 \pi-E}, m \text { integer. }
$$

But now it may seem that we made a mistake. Classically we should also have

$$
\psi_{\text {loop }}(t) \rightarrow \psi_{\text {loop }}(t+\delta t),
$$

with

$$
\delta t=-l,
$$

so that another phase factor seems to be needed:

$$
\psi_{\text {loop }} \rightarrow e^{i E l} \psi_{\text {loop }}(?)
$$

[with the same sign as Eq. (4.4)].

However, (4.8) is incorrect. The argument would only have been valid if both $\delta t$ and $E$ or $\varphi$ and $l$ could be determined independently, but instead, of course, they do not commute. It now turns out that only one factor $e^{i E l}$ as in Eq. (4.4) should be put in the boundary condition. It takes care of both the deficiency angle and the time shift, which becomes clear as soon as one either diagonalizes $E$ or $l$. The relevant question is of course what happens to the classical limit of the bulk of a wave packet, whereas its phase is meaningless in the classical limit.

There is another way to see that (4.4) takes care of everything. The energy in there is the total energy of both particles:

$$
(0, i E)=\left(\mathbf{p}_{1}, i E_{1}\right)+\left(\mathbf{p}_{2}, i E_{2}\right),
$$

where we wrote an $i$ to turn these into vectors which transform orthogonally. We can write the loop operator as

$$
e^{i l E}=e^{\varepsilon_{\mu \nu \lambda}\left(x_{1}-x_{2}\right)_{\mu} p_{1 \nu}\left(p_{1}+p_{2}\right)_{\lambda}}=e^{\varepsilon_{\mu \nu \lambda}\left(x_{1}-x_{2}\right)_{\mu} p_{1 v} p_{2 \lambda}}=e^{i l_{1} E_{2}+i E_{1} l_{2}} .
$$

Now consider particle 1 to be very heavy, and particle 2 to be a test particle. We see that the first term in the exponent in (4.10) shifts it in the time direction and the second term produces the deficiency angle. 
We conclude that the two particle problem in center of mass coordinates is completely formulated by the Schrödinger equation

$$
H=\sqrt{\mathbf{p}^{2}+m_{1}^{2}}+\sqrt{\mathbf{p}^{2}+m_{2}^{2}},
$$

for a wave function $\psi(\mathbf{r}, t)$ where $\mathbf{r}$ is on a cone with deficiency angle equal to the eigenvalue $E$ of $H$. Superposition of states with different values of $E$ will lead automatically to the required time shift on closed loops around the cone.

As stated in the introduction one can easily solve this equation by first diagonalizing $l$. One finds the anomalous quantum numbers (4.5), so that Bessel functions with non-integer indices result. But one also notes that all solutions can locally be expanded into simple plane waves. This is what we want to do with the initial and final states, so as to compute to what extent scattering occurs.

Section 5 of this paper is devoted to this simple mathematical scattering problem. It may actually have more applications than in pure $2+1$ dimensional gravity; the scattering of plane waves of whatever kind against straight sections of "cosmic strings" [4] is governed by the same equations. We will discover that indeed non-trivial scattering takes place: cosmic strings should light up when radio waves shine on them, but since we are dealing with a true interference phenomenon the effect will be far too weak to render cosmic strings directly observable this way.

Returning to the $2+1$ dimensional theory, there will still be one important step to be made: we need to know whether the two scattered particles are identical and if so, whether they satisfy Bose-Einstein or Fermi-Dirac statistics. We'll briefly return to this point in Sect. 6.

Also, as stated in the Introduction, we did not take into account the possibility that the particles annihilate each other forming a virtual graviton, which could subsequently produce a different pair. So we assume that the two particles were not each other's antiparticles. To establish whether radiative production of extra pairs might occur one has to understand the $N$ particle case better than we do now. We discuss this point further in Sect. 8.

\section{Scattering over a Branch Point}

Let us consider a flat two-dimensional space in which a wave function $\psi(\mathbf{x})$ satisfies a free-field wave equation,

$$
\left(\vec{\partial}^{2}+k^{2}\right) \psi=0
$$

with the only non-trivial feature that the origin is a branch point:

$$
\psi(\varphi+2 \pi) \neq \psi(\varphi) .
$$

At a later stage we can then replace the usual boundary condition by a conical one, identifying $\psi(\varphi)$ with $\psi(\varphi+2 \pi-\delta \varphi)$ (see later in this section).

If we restrict ourselves to a sufficiently narrow energy band then $\delta \varphi$ is welldetermined, whereas the time shift $\delta t$ across the cone will remain invisible. Indeed, if all we want is a scattering cross section we can use waves with sharply defined $k$. 


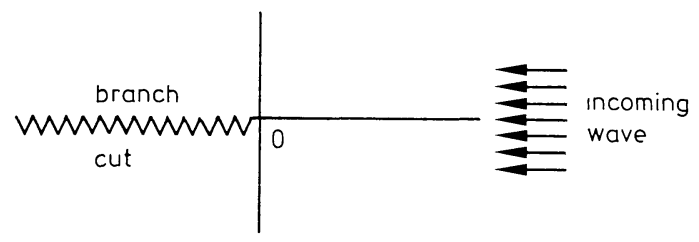

Fig. 3. Scattering against a branch cut

Consider the configuration as depicted in Fig. 3. A plane wave is entering from the right and scatters against the branch point whose cut is drawn to the left. In coordinates $r$ and $\varphi$ we now have that $\varphi$ runs from $-\infty$ to $+\infty$. The incoming wave however, at early times $t$, lives only in the region $-\pi \lesssim \varphi \lesssim+\pi$.

In cylinder coordinates the wave equation reads

$$
\partial_{r}^{2} \psi+r^{-1} \partial_{r} \psi+r^{-2} \partial_{\varphi}^{2} \psi=-k^{2} \psi .
$$

An obvious solution is

$$
\psi_{\text {in }}=e^{-i k r \cos \varphi},
$$

for all $r$ and $\varphi$.

We want the incoming wave to be like Eq. (5.4), but only in the region

$$
-\pi \lesssim \varphi \lesssim \pi ; r \rightarrow \infty
$$

we will now look for a solution of (5.3) that approaches (5.4) in the region (5.5) but goes to zero in the region

$$
|\varphi|>\pi ; r \rightarrow \infty .
$$

This exercise is easier than one might think.

Let us rewrite (5.4) as

$$
\oint_{c} \frac{d \sigma}{2 \pi i(\sigma-\varphi i)} e^{-i k r \cosh \sigma},
$$

where the contour $C$ just runs around the pole at $\sigma=\varphi i$. Of course one can check that (5.7) satisfies (5.3), by doing partial integration. However, this proof does not depend on the exact location of the contour. Indeed, we could put the contour somewhere else, and get an equally valid solution. The contour we are now interested in is given in Fig. 4. It is a double contour, $C=C_{1}+C_{2}$. At the end points the integrand oscillates rapidly and tends to zero.

At $r \rightarrow \infty$ the contribution of the horizontal sections of both $C_{1}$ and $C_{2}$ can easily be seen to vanish. So then the contour closes, and we recover (5.7), but only when $-\pi<\varphi<\pi$. If $|\varphi|>\pi$ the contour closes with the pole outside and hence the integral vanishes. And so we found the solution of (5.3) that satisfies the required boundary conditions in (5.5) and (5.6):

$$
\psi(\mathbf{x})=\int_{C_{1}+C_{2}} \frac{d \sigma}{2 \pi i(\sigma-\varphi i)} e^{-i k r \cosh \sigma} .
$$




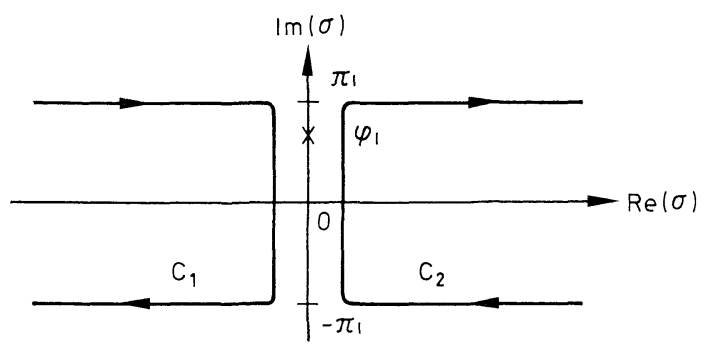

Fig. 4. Location of the contour $C=C_{1}+C_{2}$ in Eq. (5.8)

We can now rearrange $C_{1}$ and $C_{2}$ together in three pieces, two horizontal lines from $-\infty$ to $\infty$ at $\operatorname{Im}(\sigma)= \pm \pi$, and a closed contour around the pole, which contributes only if $|\varphi|<\pi$. One then obtains

$$
\psi(r, \varphi)=e^{-i k r \cos \varphi} \theta(\pi-|\varphi|)+\psi_{1}(r, \varphi-\pi)-\psi_{1}(r, \varphi+\pi),
$$

where

$$
\psi_{1}(r, \varphi)=\int_{-\infty}^{\infty} \frac{d \sigma}{2 \pi i(\sigma-i \varphi)} e^{i k r \cosh \sigma} .
$$

By construction, (5.9) is a continuous function of $r$ and $\varphi$.

The first part of (5.9) represents the incoming wave, as well as that part of the wave that continues in the forward direction without being scattered. $\psi_{1}$ represents the scattered waves. For large $r$ we can expand

$$
\cosh \sigma \rightarrow 1+\sigma^{2} / 2
$$

and we find

$$
\psi_{1}(r, \varphi) \rightarrow \frac{e^{i k r}}{\varphi \sqrt{-2 \pi i k r}}
$$

Note that this is a nearly spherical wave, and that the intensity of scattered particles per unit angle is

$$
J(\varphi)=[1 /(\varphi-\pi)-1 /(\varphi+\pi)]^{2} /(2 \pi k),
$$

which for large angles goes like $2 \pi /\left(k \varphi^{4}\right)$. Thus the particles can wrap around the origin many times, but with a rapidly decreasing probability. This is the process we referred to in the Introduction.

Next, we consider scattering over a wedge. Suppose that now

$$
\psi(\varphi+2 \pi \alpha)=\psi(\varphi)
$$

with

$$
2 \pi \alpha=2 \pi-\delta \varphi=2 \pi-E .
$$

Then we sum expression (5.9) over the $\varphi$ values

$$
\varphi_{n}=\varphi+2 \pi \alpha n, \quad n=-\infty, \ldots, \infty .
$$




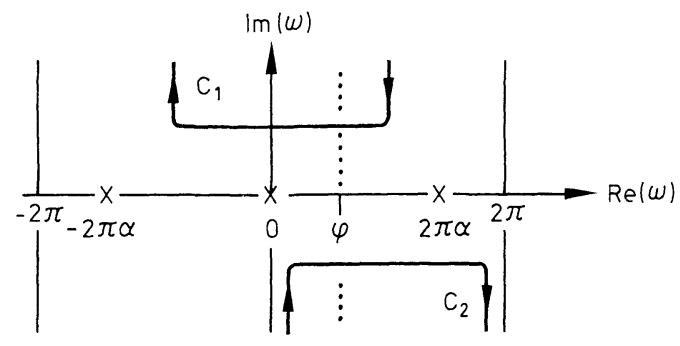

Fig. 5. Contours $C_{1}$ and $C_{2}$ in the integral (5.19)

Thus we obtain

$$
\psi_{\alpha}(r, \varphi)=\sum_{n=-\infty}^{\infty} e^{-i k r \cos (\varphi+2 \pi n \alpha)} \theta(\pi-|\varphi+2 \pi n \alpha|)+\psi_{1 \alpha}(r, \varphi-\pi)-\psi_{1 \alpha}(r, \varphi+\pi),
$$

with

$$
\psi_{1 \alpha}(r, \varphi)=\sum_{n=-\infty}^{\infty} \int_{-\infty}^{\infty} \frac{d \sigma}{2 \pi(\varphi+2 \pi n \alpha+i \sigma)} e^{i k r \cosh \sigma}=\int_{-\infty}^{\infty} d \sigma \frac{e^{i k r \cosh \sigma}}{4 \pi \alpha \operatorname{tg}\left(\frac{\varphi+i \sigma}{2 \alpha}\right)}
$$

When $r$ tends to infinity we find the scattering amplitude,

$$
\begin{aligned}
f(\varphi)= & \sqrt{r}\left[\psi_{1 \alpha}(r, \varphi-\pi)-\psi_{1 \alpha}(r, \varphi+\pi)\right] e^{-i k r} \\
& =\frac{1}{2 \alpha \sqrt{-2 \pi i k}}(\cot [(\varphi-\pi) / 2 \alpha]-\cot [(\varphi+\pi) / 2 \alpha]) .
\end{aligned}
$$

Note that $\psi_{\alpha}$ (Eq. 5.16) can also be written as a contour integral,

$$
\psi_{\alpha}(r, \varphi)=\int_{C_{1}+C_{2}} \frac{-i d \omega}{4 \pi \alpha \operatorname{tg}(\omega / 2 \alpha)} e^{-i k r \cos (\varphi-\omega)},
$$

where the contours $C_{1}$ and $C_{2}$ are the ones depicted in Fig. 5.

We regard the first part of Eq. (5.16) as the unperturbed or forward wave, and conclude that $\psi_{1 \alpha}$ represents non-trivial quantum mechanical scattering.

It is important to note that in our derivation of the scattering amplitude $f(\varphi)$ we did not need to know how the Hamiltonian (4.11) depends on the Laplacian $k^{2}$. This is because we used wave packets whose $k$ value is (practically) fixed. Even the group velocity, determined by the first derivative, $\partial k / \partial p$, was not needed. Hence the result is exact even if the relativistic Hamiltonian (4.11) is used. This is a rather special feature of this system, where all dynamics comes from the space-time topology and there are no potentials.

\section{Statistics}

The previous section produced the scattering amplitude for the case that the two particles were not identical. If they are then we have to realize that we should limit 
ourselves to states that are either symmetric (Bose-Einstein) or antisymmetric (Fermi-Dirac) under interchange of the two particles. In Eq. (3.1) this corresponds to interchanging $\mathbf{x}_{1}$ and $\mathbf{x}_{2}$. This interchange operator will produce a state that interferes with that part of the wave function in which particles 1 and 2 rotated to their new position, in either direction. In both cases, one of the two cusps passes over the detector $\mathbf{0}$, in either direction. Since the two cusps are now equal, this means that the interchange is associated with the square root of the operator $P_{2} P_{1}$ of Eq. (3.3). Thus, the interchange operator will link $\psi(\mathbf{r})$ with $\psi\left(\mathbf{r}^{\prime}\right)$, where $\mathbf{r}^{\prime}$ is a point on the cone diametrically opposite to $\mathbf{r}$, or

$$
\left(r^{\prime}, \varphi^{\prime}\right)=(r, \varphi+\pi-\delta \varphi / 2),
$$

if $\delta \varphi=E$ is the deficiency angle.

The prescription is now simple: if we are dealing with identical particles then our incoming wave must be chosen symmetric or antisymmetric under the interchange (6.1), and then automatically the scattered wave will exhibit the same symmetry. Consequently, both $\psi_{\alpha}$ and $\psi_{1 \alpha}$ in the previous sector (and therefore also the scattering amplitude $f$ ) will have to be replaced as follows:

$$
\psi(r, \varphi) \rightarrow \psi(r, \varphi) \pm \psi(r, \varphi+\alpha \pi) .
$$

As for the time shift we note that the particle interchange operator will be accompanied by the operator

$$
e^{i l E / 2},
$$

so that, as before, the time shift is accommodated for automatically.

\section{The $N$ Particle Case}

As stated before, extension of our results to the $N$ particle case is somewhat enigmatic. We can't resist saying something more about it; the problem seems to be a beautiful one. But we stress that the approach indicated in this section is preliminary and incomplete.

We may consider first extending the $N$ particle Hilbert space in an ordinary flat two-dimensional space (at a given time) by "unfolding" it: two states obtained from each other by rotating one particle in a closed loop of $2 \pi$ radians around another (without enclosing other particles) are to be considered different. The best way to indicate this difference is by attaching strings to the particles. Not the fine details of these strings but only the topology of the way they go between other particles is a relevant extra "degree of freedom" of our system.

Later we will have to remove these string degrees of freedom by identifying states with different string topologies using our looping operators, but let us for a moment concentrate on our extended Hilbert space.

It is very large. In the two particle case it is easiest to use cylinder coordinates. We then see that the angle $\varphi$ runs from $-\infty$ to $\infty$ instead of $-\pi$ to $\pi$. The relative angular momentum would be continuous rather than quantized. In the $N$ particle case the situation is even worse. Particles 1 and 2 may twist around each other $n_{1}$ times, then particles 2 and 3 may whirl around each other $n_{2}$ times, then 1 and $3 n_{3}$ 
times, and so on. Indeed, the strings may form braids of arbitrary length with $N$ strands: knot theory seems to be relevant for this Hilbert space!

In this Hilbert space we wish to define the operators $x_{i_{\mu}}$ and $p_{i_{\mu}}$, where the index $i$ refers to particle number $i$ and the Greek indices $\mu, v, \ldots$ are Lorentz indices. Note that apart from the many Riemann sheets, we may use ordinary flat coordinates, so the spacelike components, $x_{i_{1}}$ and $x_{i_{2}}$, are well defined. $x_{i_{3}}$ are all equal to $i t$, where $t$ is one time coordinate and $i=\sqrt{-1}$.

We can also define $p_{i_{1,2}}$ as differentiations with respect to $x_{i_{1}, 2}$, provided that the differentiation can be done also at the branch points. Let us assume that all wave functions are restricted to be $C_{\infty}$, also at the branch points (so that at the branch points themselves all Riemann sheets merge smoothly), a condition that we can later perhaps relax.

It remains to define the operators $p_{i_{3}}=i p_{i_{0}}$. These are more tricky. The mass shell condition reads

$$
p_{i_{0}}=+\sqrt{p_{i}^{2}+m_{i}^{2}} .
$$

Is this a well-defined operator in our extended Hilbert space? In the two particle case there seemed to be no problem in choosing the positive sign since we chose to work with eigenvectors of the energy operator $E$. Let us assume that (7.1) is a reasonable definition.

We are then in a position to define the looping operators. Associated with each pair of particles $i$ and $j$ we have

$$
S_{i j}=e^{\varepsilon_{\mu \nu \lambda}\left(x_{i}-x_{j}\right)_{\mu} p_{\nu \nu} p_{j \lambda} \lambda} .
$$

In addition, we have the "string knot" operator, $T_{i j}$ which produces one extra knot between strings $i$ and $j$ without involving the other particles. This means that the knot must have been produced by rotating one particle around the other without enclosing a third. The direction of the rotation (the "sign" of the knot) is defined in accordance with the previous sections. We now remove the redundant degrees of freedom by postulating that

$$
S_{i j} T_{i j}|\psi\rangle=|\psi\rangle, \text { for all } i, j .
$$

This condition is derived using the same methods as in Sects. 3 and 4; all particles other than the $i$ th and $j$ th are treated just as the observer $\mathbf{0}$ in Sect. 3 .

It is difficult to understand precisely the consequences of condition (7.3), indeed whether an $N>2$ particle Hilbert space satisfying it exists at all. Classical analysis suggests that if the total energy exceeds $2 \pi$ then space is made compact. Apparently then, Eq. (7.3) identifies infinitely many points in 2 -space. As yet it remains a conjecture that Eq. (7.3) defines non-perturbatively a $2+1$ dimensional quantum theory of gravitating particles.

\section{Comparison with Perturbative Gravity}

To split Hilbert space first into sectors with definite numbers of particles $(N)$ was an important step in our approach. In the rudimentary discussion of the general $N$ particle case as given in the previous section it seemed that these sectors are 
independent, because no creation or annihilation take place. The possibility to define the operators $p_{i_{0}}$ [Eq. (7.1)] with the plus sign suggests that there are no transitions between positive and negative frequencies, so that there is no role for antiparticles.

This now sounds unlikely. Let us briefly consider perturbative quantum gravity in $2+1$ dimensions. The propagator is

$$
P_{\mu \nu \alpha \beta}=\frac{\eta_{\mu \alpha} \eta_{\nu \beta}+\eta_{v \alpha} \eta_{\mu \beta}-2 \eta_{\mu \nu} \eta_{\alpha \beta}}{k^{2}-i \varepsilon},
$$

where $\eta_{\mu \nu}=\operatorname{diag}(1,1,1,-1)$. Let us rotate $k_{\mu}$ into

$$
k_{\mu}=\left(\begin{array}{c}
0 \\
k_{3} \\
k_{0}
\end{array}\right) \text {, }
$$

and define

$$
\bar{k}_{\mu}=\left(\begin{array}{c}
0 \\
k_{3} \\
-k_{0}
\end{array}\right) ; \quad \hat{k}_{\mu}=\left(\begin{array}{c}
k_{3} \\
0 \\
0
\end{array}\right)
$$

Then one can write

$$
\eta_{\mu \nu}=\frac{1}{2 k_{3}^{2}}\left(k_{\mu} \bar{k}_{v}+\bar{k}_{\mu} k_{v}-2 k^{2} \delta_{\mu 0} \delta_{v 0}+2 \widehat{k}_{\mu} \hat{k}_{v}\right),
$$

and one finds that the propagator splits into two parts:

$$
P_{\mu \nu \alpha \beta}=P_{\mu \nu \alpha \beta}^{(1)}+P_{\mu \nu \alpha \beta}^{(2)},
$$

with

$$
\begin{aligned}
P_{\mu \nu \alpha \beta}^{(1)}= & \frac{1}{4\left(k^{2}-i \varepsilon\right) k_{3}^{4}}\left(2 k_{\mu} k_{v} \bar{k}_{\alpha} \bar{k}_{\beta}+2 \bar{k}_{\mu} \bar{k}_{v} k_{\alpha} k_{\beta}-\left(k_{\mu} \bar{k}_{v}+\bar{k}_{\mu} k_{v}\right)\left(k_{\alpha} \bar{k}_{\beta}+\bar{k}_{\alpha} k_{\beta}\right)\right. \\
& \left.+k_{\mu} Q_{v \mid \alpha \beta}+k_{\nu} Q_{\mu \mid \alpha \beta}+k_{\alpha} Q_{\beta \mid \mu \nu}+k_{\beta} Q_{\alpha \mid \mu \nu}\right),
\end{aligned}
$$

where

$$
Q_{\mu \mid \alpha \beta}=k^{2}\left[4 \bar{k}_{\mu} \delta_{\alpha 0} \delta_{\beta 0}-2 \bar{k}_{\alpha} \delta_{\mu 0} \delta_{\beta 0}-2 \bar{k}_{\beta} \delta_{\mu 0} \delta_{\alpha 0}\right]-4 \bar{k}_{\mu} \hat{k}_{\alpha} \hat{k}_{\beta}+2 \hat{k}_{\mu}\left(\hat{k}_{\alpha} \bar{k}_{\beta}+\hat{k}_{\beta} \bar{k}_{\alpha}\right) ;
$$

and

$$
\begin{aligned}
P_{\mu \nu \alpha \beta}^{(2)}= & \frac{1}{k_{3}^{4}}\left(2 \delta_{\mu 0} \delta_{v 0} \widehat{k}_{\alpha} \hat{k}_{\beta}+2 \delta_{\alpha 0} \delta_{\beta 0} \widehat{k}_{\mu} \widehat{k}_{v}-\delta_{\mu 0} \delta_{\alpha 0} \widehat{k}_{v} \hat{k}_{\beta}-\delta_{v 0} \delta_{\alpha 0} \widehat{k}_{\mu} \widehat{k}_{\beta}\right. \\
& \left.-\delta_{\mu 0} \delta_{\beta 0} \hat{k}_{v} \hat{k}_{\alpha}-\delta_{v 0} \delta_{\beta 0} \hat{k}_{\mu} \hat{k}_{\alpha}\right) .
\end{aligned}
$$

Now the simplest graviton scalar particle vertex as pictured in Fig. 6 is

$$
R_{\mu \nu}=\eta_{\mu v}\left((p q)-m^{2}\right)-p_{\mu} q_{v}-p_{v} q_{\mu},
$$




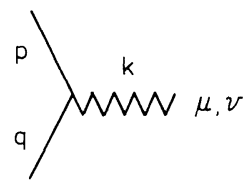

Fig. 6. The scalar - graviton vertex ( $p, q$, and $k$ are momenta; $p+q+k=0)$

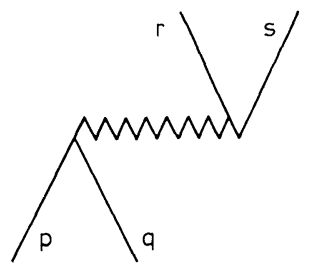

Fig. 7. Pair amnihilation and creation

which satisfies

$$
k_{\mu} R_{\mu \nu}=\left(m^{2}+p^{2}\right) q_{v}+\left(m^{2}+q^{2}\right) p_{v},
$$

which vanishes on mass shell (this of course follows from energy-momentum conservation).

As a consequence, the part $P_{\mu \nu \alpha \beta}^{(1)}$ of the propagator does not contribute in a scattering diagram. The part $P_{\mu \nu \alpha \beta}^{(2)}$ has no $1 /\left(k^{2}-i \varepsilon\right)$ pole; it represents instantaneous gravitational interaction without any real gravitons being transmitted. Of course this confirms that there are no gravitons. We expect that $P^{(2)}$ reproduces the scattering as we derived it in the previous sections.

However, there is also creation and annihilation. Consider the diagram of Fig. 7. If we go to the center of mass frame, writing

$$
p_{\mu}=\left(\begin{array}{c}
0 \\
p_{3} \\
p_{0}
\end{array}\right) ; \quad q_{\mu}=\left(\begin{array}{c}
0 \\
-p_{3} \\
p_{0}
\end{array}\right),
$$

we find for the incoming particles on mass shell

$$
R_{\mu \nu}=\operatorname{diag}\left(-2 p_{0}^{2},-2 m^{2}, 0\right) \equiv A(\mu) \delta_{\mu \nu},
$$

and, in the case that the created particles either move parallel or orthogonally to the incoming ones we have a similar expression,

$$
R_{\mu \nu}=B(\mu) \delta_{\mu \nu}
$$

for the created particles.

Plugging our propagator (8.1) between these vertices we get the amplitude

$$
-2 A_{1} B_{2}-2 A_{2} B_{1}
$$

which always keeps the same sign and therefore cannot vanish. Thus, perturbation theory does predict creation and annilation. More generally, one expects radiative 


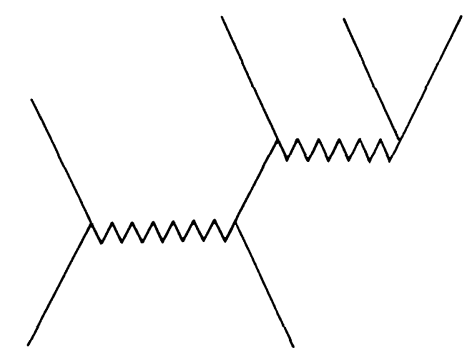

Fig. 8. Radiative production

pair production via diagrams such as Fig. 8, even if we started with scattering particles that are not each other's anti-particles.

Therefore, we suspect that a more complete formulation of the $N$ particle system might reveal difficulties with the sign choice in (7.1), but we haven't found out what they are. At first sight the operators $S_{i j}$ [Eq. (7.2)] just generate elements of the homogeneous Lorentz group, which would leave the sign intact. It could be that the operators $T_{i j}$ (which in a subtle way depend on the locations of the other particles), in particular the choice of their phase, will give trouble.

Quite independent of our interpretation of the $2+1$ dimensional theory of quantum gravity is our method to solve the scattering problem in Sect. 5. As said before, this method may have other applications.

Acknowledgements. The author benefitted from many discussions, notably with $\mathrm{H}$. van Dam, R. Jackiw, S. Deser, C. Korthals Altes, and P. van Baal.

$\mathrm{He}$ also thanks Princeton University and the Institute for Advanced Study for their hospitality; part of this work was supported by grant number PHY-8620266.

\section{References}

1. Gott III, J.R., Alpert, M.: General relativity in a $(2+1)$ dimensional space-time. Gen. Rel. Grav. 16, 243 (1984)

Staruszkiewicz, A.: Gravitation theory in three-dimensional space. Acta Phys. Polon. 24, 734 (1963)

Deser, S., Jackiw, R., 't Hooft, G.: Three dimensional Einstein gravity: dynamics of flat space. Ann. Phys. 152, 220 (1984)

Giddings, S., Abbott, J., Kuchar, K.: Einstein's theory in a three-dimensional space-time. Gen. Rel. Grav. 16, 751 (1984)

2. Jackiw, R.: Private communication

3. DeWitt, B.S.: Phys. Rev. Lett. 12, 742 (1964); Quantum theory of gravity. I. Phys. Rev. 160, 1113 (1967); II, III. Phys. Rev. 162, 1195, 1239 (1967)

't Hooft, G., Veltman, M.: One loop divergencies in the theory of gravitation. Ann. Inst. Henri Poincaré 20, 69 (1974)

4. Kibble, T.W.B.: Topology of cosmic domains and strings. J. Phys. A 9, 1387 (1976)

Vilenkin, A.: Gravitational field of vacuum domain walls and strings. Phys. Rev. D 23, 852 (1981); Cosmic strings and domain walls. Phys. Rep. 121, 263 (1985) 